\title{
Supporting Information for: Nanowire probes for high resolution combined scanning electrochemical microscopy - atomic force microscopy
}

\section{Thermal Oxidation of Silicon Probes}

Silicon AFM probes (Force Modulation MULTI75 Lot Oriel) were thermally oxidised dry in a 1 inch tube furnace oven on a quartz boat under an $\mathrm{O}_{2}$ flow of 200 standard cubic centimetres per minute (sccm) for a total of 9 hours ( 3 runs for 3 hours) at $1100{ }^{\circ} \mathrm{C}$. Oxidised probes were cooled slowly under oxygen between runs. Gas flow rates were accurately controlled using mass flow controllers. Figure 1 shows a FE-SEM cross section image clearly showing the $460 \mathrm{~nm}$ thick thermal oxide film which provides a pin-hole free insulating layer on the silicon. Cyclic voltammetry (Figure 2) performed on an oxidised cantilever in a solution containing $10 \mathrm{mM} \mathrm{IrCl}_{6}{ }^{3-}$ and $0.1 \mathrm{M} \mathrm{KNO}_{3}$, displayed only very low capacitive currents, indicating a well-insulated film.

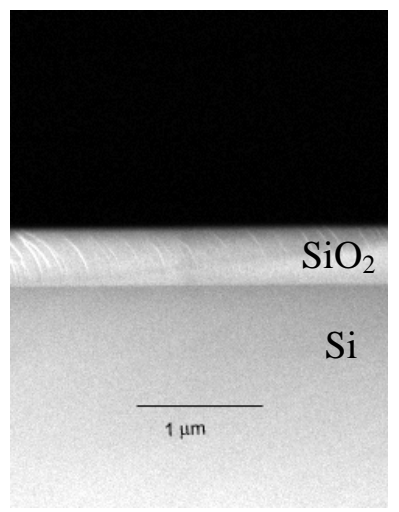

Figure 1. FE-SEM cross-section image of a thermally oxidised silicon AFM probe. 


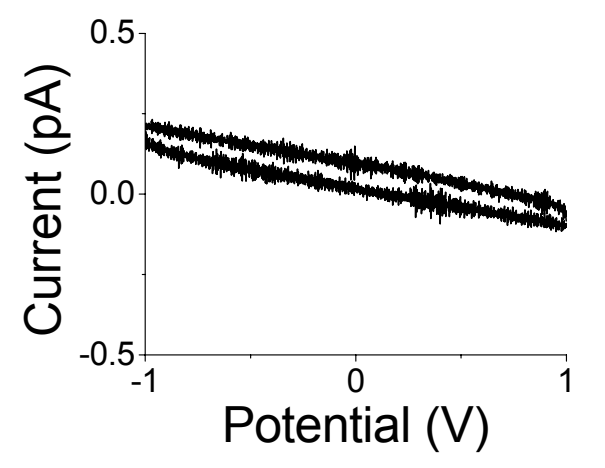

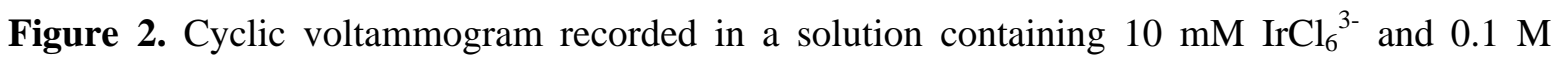
$\mathrm{KNO}_{3}$ at a scan rate of $20 \mathrm{mV} \mathrm{s}^{-1}$, using a silicon AFM probe (FESP, L. O. T. -Oriel Gmbh \& Co.) which had been thermally oxidised for 3 hours by the procedure described above, as the working electrode. $\mathrm{An} \mathrm{Ag} / \mathrm{AgCl}$ reference electrode was employed.

\section{Poly(oxyphenylene) Insulation Layer}

A thin conformal poly(oxyphenylene) film was formed on the Au-coated single walled carbon nanotube bundle AFM tip by the electropolymerisation of a 1:1 methanol:water-based monomer solution containing $60 \mathrm{mM}$ phenol, $90 \mathrm{mM}$ 2-allylphenol and $160 \mathrm{mM}$ 2-n-butoxyethanol. The solution $\mathrm{pH}$ was adjusted to 9.2 by the drop wise addition of ammonium hydroxide and used on the same day as preparation. Electropolymerization was conducted at a potential of $+2.5 \mathrm{~V}$ against a silver wire quasi-reference electrode (AgQRE) for 20 minutes. The probe was positioned so that the tip apex faced the reference electrode. The probe was carefully rinsed in a 1:1 methanol:water solution immediately after electrodeposition. The copolymer film was subsequently heat cured at $150{ }^{\circ} \mathrm{C}$ for 30 minutes to form a pinhole free insulating layer.

\section{Silicon Nitride Insulation Layer}

Silicon nitride was deposited on either side of the probe, which served as a protective layer to prevent salt damage and prolong the lifetime and stability of the probe.

Silicon nitride films were deposited on the insulated nanowire probes using a room-temperature deposition technique, namely inductively coupled plasma chemical vapor deposition (ICP CVD) 
employing $\mathrm{SiH}_{4} / \mathrm{N}_{2}\left(\mathrm{NH}_{3}\right.$ free: Plasmalab System 100 ICP180 from Oxford Instruments Plasma Technology).

\section{Focused Ion Beam (FIB) Cutting}

Focused ion beam milling was achieved using a FEI Strata FIB200TEM system. During probe alignment, prior to nanowire cutting, single scans were used with a low beam current to minimize damage to the insulating layer. The insulated nanowire was cut using a beam current of $\sim 10 \mathrm{pA}$. Care was taken to ensure a good electrical contact between the probe and the FIB sample holder, to minimised local charging at the tip apex.

\section{Topographical Imaging with the nanoelectrode probes:}

To demonstrate the high topographic resolution possible with the nanowire based SECM-AFM probes, a $1 \mu \mathrm{m}$ square height image of evaporated Au on a silicon substrate was recorded (Figure 3). The image was taken in tapping mode, in air (height scale $15 \mathrm{~nm}$ ).

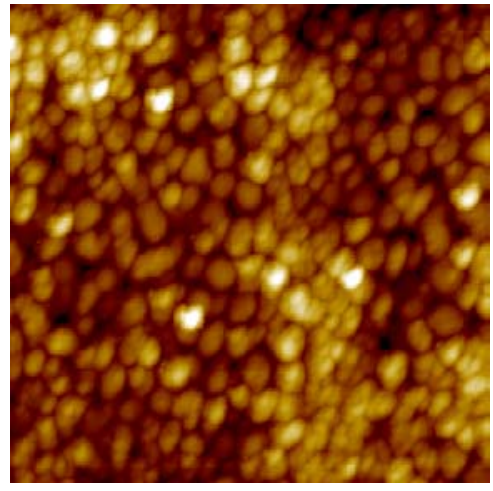

Figure 3. $1 \mu \mathrm{m}$ square height image of Au on a silicon substrate (15 $\mathrm{nm}$ height scale). 